\title{
Fouling of Flow Reactors in Organolithium Mediated Transformations: Experience on Scale-up and Proposed Solution
}

\author{
Paolo Filipponi, Bertrand Guelat, Julien Haber, Serena Mostarda, Ruairi O’Meadhra, Lorenzo \\ Piccioni, Jutta Polenk, Berthold Schenkel, Silke Schoenebeck, Alexander Streit, Roger Suremann, \\ Francesco Venturoni ${ }^{\star}$, and Stefan Wegmann
}

\begin{abstract}
Continuous processing has been demonstrated to be a superior approach when applied to fast and energetic chemical transformations. Indeed, whereas classical batch or semi-batch methods require cryogenic conditions and slow addition rates of reactive species, flow technologies enable rapid mixing of synthetic partners in a highly controlled environment. As a result, low yielding and dangerous processes in batch can be performed at scale in a cost competitive and safer continuous manner. Despite the advantages of higher quality and safety, the perennial problems of solids build-up and pipe fouling threaten the robustness and reliability of flow processes. In this contribution, a new methodology to prevent reactor fouling is reported and discussed. The implementation of this methodology has been decisive in solving fouling issues encountered during the piloting of an organolithium based flow process.
\end{abstract}

Keywords: Avoid clogging · Continuous manufacturing · Flow chemistry · Fouling · Organolithium reagents

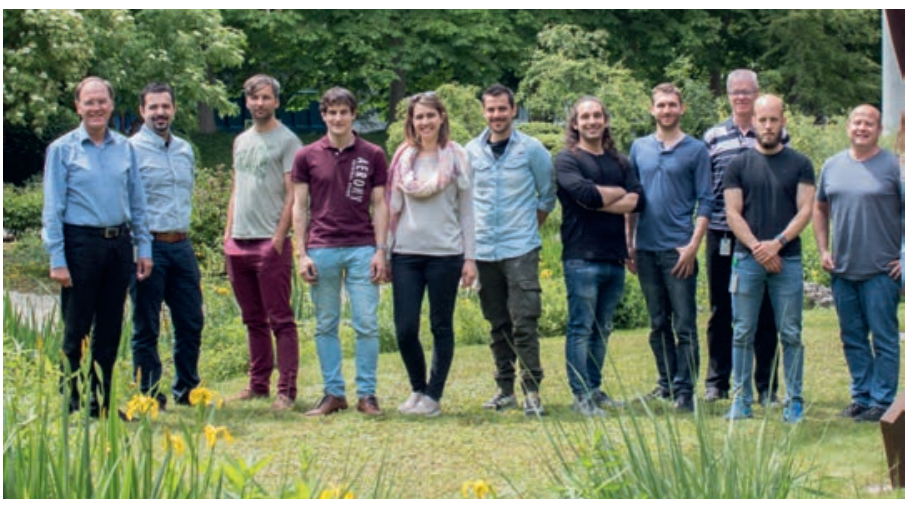

The Novartis continuous manufacturing unit (upstream). Left to right: Berthold Schenkel (team leader), Francesco Venturoni (chemist), Julien Haber (chemical engineer), Bertrand Guelat (chemical engineer), Serena Mostarda (chemist), Paolo Filipponi (chemist), Lorenzo Piccioni (scientist), Alexander Streit (scientist), Ruairi O'Meadhra (chemical engineer), Stefan Wegmann (scientist), Roger Suremann (scientist), missing from the picture: Jutta Polenk and Silke Schoenebeck.

\section{Introduction}

The changing face of chemical manufacturing has a direct impact on the way in which molecules are made. While the assembly of chemical entities by formation and disconnection of new bonds is still the ultimate goal, atom-efficiency, process robustness, analytical quality and manufacturing costs of chemical processes have become the main focus. High and rising standards in quality and cost effectiveness are increasing the pressure on chemical

${ }^{\star}$ Correspondence: Dr. F. Venturoni, E-mail: francesco.venturoni@novartis.com Novartis Pharma AG, Chemical and Analytical Development, WKL-122, $\mathrm{CH}-4002$ Basel production. ${ }^{[1]}$ Within this framework, flow processing enables an increasing number of synthetic options, with flexibility in pressure, temperature and reagent selection, as well as permitting hazardous chemistries under well-defined conditions. ${ }^{[2]}$ The hidden potential of the so-called enabling technologies is nowadays unleashed, reshaping both synthesis schemes and manufacturing plants. Among the enabling technologies, flow chemistry, which has been an established tool in the bulk chemical industries, finds more and more applications in the fine chemical and pharmaceutical productions. ${ }^{[3]}$ Its quality and cost impact are systematically compared to the existing batch manufacturing methods. ${ }^{[3]}$ In this context, leveraging of flow chemistry in organometallic transformation has attracted a great deal of attention in the past few years in both academia and industry. Indeed, flow chemistry allows the maintenance of well-defined conditions such as temperature, reaction time and mixing time, which have been found beneficial for the handling of unstable organolithium intermediates. ${ }^{[4,5]}$ The competition with batch or semi-batch approach is not relevant especially when dealing with the scale-up of fast and highly energetic reactions. In these cases, a semi-batch approach in which one reagent is dosed on to another reagent, precooled to the desired temperature, is the common way to achieve comparable conditions in batch facilities. The addition rate is used as parameter for temperature control, and as a consequence an extended period of dosage can be expected. In these circumstances, it can be the case that the temperature reached during the addition combined with the addition rate is not compatible with the half-life of the organolithium intermediate leading to scale-up issues and unstable processes. Although the scale-up of the above described class of reactions in continuous flow is chemically convenient, it also presents some challenges that discourage the application of this technology at manufacturing scale. These include the fouling of continuous tubular reactors which may lead to complete blockage and interruption of the chemical process. ${ }^{[6,7]}$ In particular, while working with organolithium reagents, three main causes of foul- 
ing have been reported such as the precipitation of lithium intermediate, the precipitation of the substrate/reagent/product (this should not be the case in late-stage process development) and the formation of $\mathrm{LiOH}$ deposits from water present in solvents. Regarding the first two scenarios, process development activities such as solvent selection, concentration adjustments and additives screening, may be instrumental in avoiding reactor fouling. In the present manuscript, the activities to avoid the formation and precipitation of $\mathrm{LiOH}$ are reported and discussed.

\section{Results and Discussion}

\section{Fouling Experience in the Pilot Plant}

The preparation of a Novartis product involves as the key step the reaction of an arylbromide derivative with hexyllithium (HexLi) under flow conditions at $-30{ }^{\circ} \mathrm{C}$. The process was piloted in two different facilities both with a throughput of $c a .2 .5 \mathrm{~kg}$ of final compound per hour. Both facilities utilized a tubular reactor and static mixer to facilitate the mixing of the streams. During the piloting phase, it was reported that the process had to be stopped and the reactor cleaned because of pressure increase due to fouling of the reactor. Depending on the reactor dimensions, the quality of the starting material and solvent type, the run times ranged between 9 and $30 \mathrm{~h}$ before encountering a blockage. In one of the pilot technical batches the reactor was disassembled after pressure increase. The reactor walls were visually inspected and a layer of white solid was observed. Powder diffraction analysis and IR of the solid indicated that the material was composed mainly of $\mathrm{LiOH}$ (Fig. 1), which could be identified as the main cause for the repeated plugging. It is mainly formed from the reaction between $\mathrm{HexLi}$ and the moisture contained in the starting material feed solution.

After the unexpected fouling issues in pilot scale a lab investigation aimed at mitigating the reactor fouling caused by $\mathrm{LiOH}$ was initiated with the perspective to reduce the number of process interruptions at commercial scale.

\section{Process Development in the Flow Lab Unit}

Prior to the piloting runs described above, the plant process had been developed using a set-up composed of two micro an- nular pumps (HNP), which feed the flow reactor with a solution of starting material (0.3 M in THF) and HexLi (2.3 $\mathrm{M}$ in hexane) respectively at $20 \mathrm{ml} / \mathrm{min}$ of total flow rate to guarantee fast mixing (Fig. 2).

The starting material solution was pre-cooled in-line before mixing at $-30{ }^{\circ} \mathrm{C}$ with a $1 / 8$ inch loop. The HexLi stream was pre-cooled at $0{ }^{\circ} \mathrm{C}$ since lower temperature would increase the risk of solids precipitation from the solution. The mixing element consisted of a T-mixer with internal diameter of $0.5 \mathrm{~mm}$, ensuring fast mixing at the given flow rate. ${ }^{[8]}$ The mixing section was followed by $1 / 16$ inch tube with a length adjusted to meet the desired residence time. Under these conditions, the flow process had been run for more than $6 \mathrm{~h}$ without encountering any issues.

\section{Fouling Characterization in the Flow Lab Unit}

When trying to reproduce the fouling issues on the laboratory set-up as described above, it was quickly clear that the results generated under these conditions were not fully reproducible. The time at which fouling caused a complete clogging of the reactor varied up to $\pm 100 \%$ from experiment to experiment, under the same conditions. An evaluation of an appropriate lab set-up to study the fouling was then conducted. A commercially available Ehrfeld FlowPlate Lab reactor ${ }^{[9]}$ was selected, equipped with the following plate 'SZ-Mischer, Nennweite 0,5 mm, Multi-injection 3, Version 2'. The transparent glass plate at the top of the reactor allowed a high-resolution camera to be installed to monitor the reaction outcome and the formation of solids in the reactor. The Ehrfeld reactor is composed of mixing and residence time zones, whose respective geometries provide different mixing and residence time characteristics. The mixing zone has narrow tortuous passages to promote streamline folding at higher velocity thus ensuring fast mixing times. The residence time zone has straight wider section to prolong holding time on the plate (Fig. 3).

\section{Comparison of Laboratory and Pilot Scale Equipment}

For the scale down of the process from pilot scale into the Ehrfeld reactor, the flow rate was selected such that sufficiently

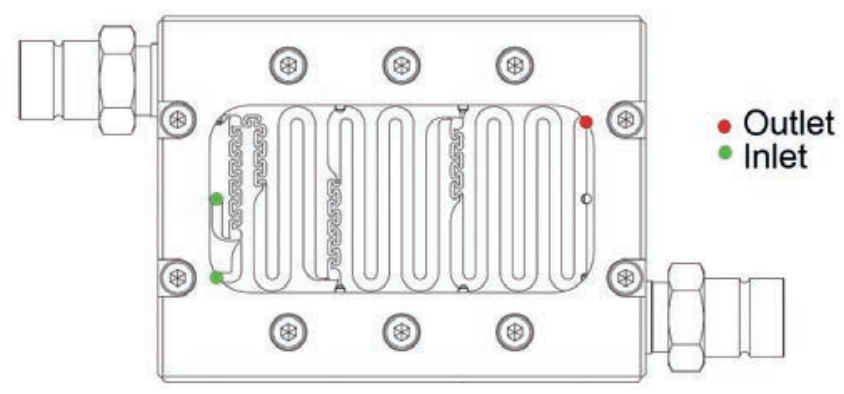

Fig. 3. Ehrfeld reactor scheme used to investigate solid build-up and
Fig. 1. Pictures of the inside of the reactor tubes taken with a video probe (GE Mentor Visual IQ): Static mixer coated by LiOH (left), reactor wall coated by $\mathrm{LiOH}$ (right). fouling.

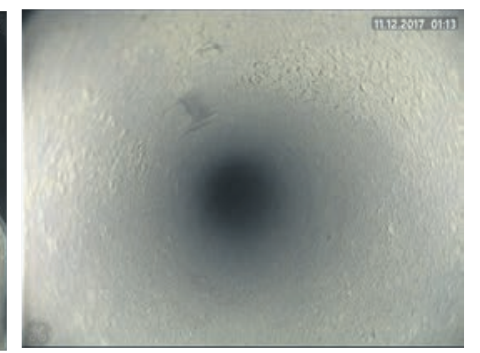

Fig. 2. Flow scheme and set-up used in process development.

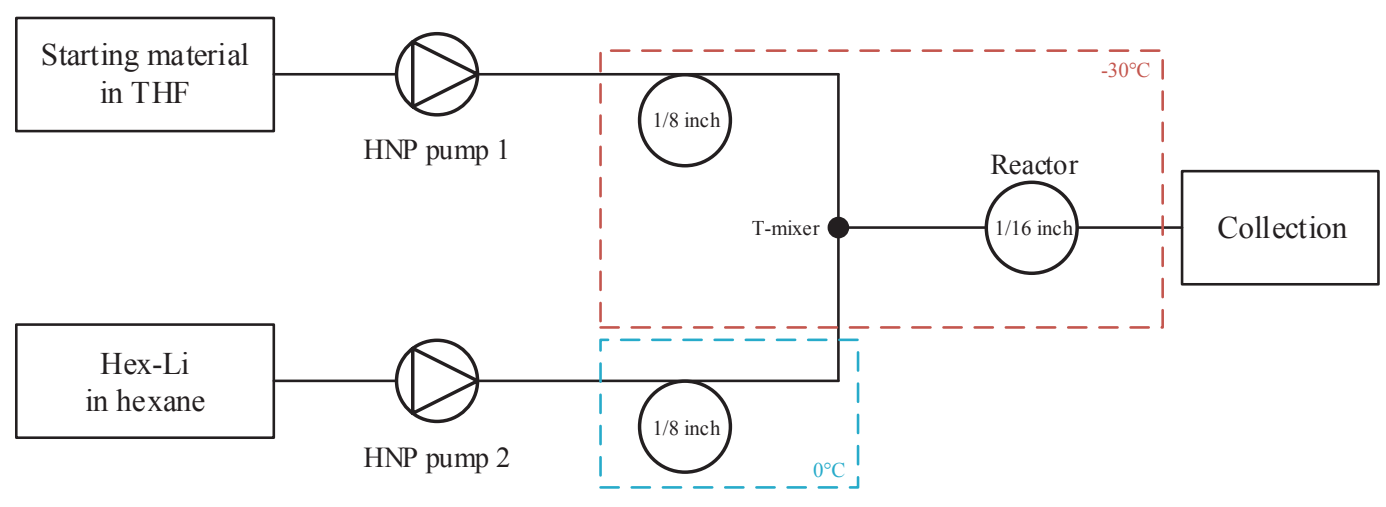


Table 1. Comparison between typical laboratory and pilot production scale conditions

\begin{tabular}{|l|l|l|}
\hline Item & $\begin{array}{l}\text { Ehrfeld Lab Scale } \\
\text { Mixing zone / RT zone }\end{array}$ & Pilot Production Scale \\
\hline Internal diameter $[\mathrm{mm}]$ & $1.25 * 0.50 / 2.5 * 1$ & 6 \\
\hline Cross section $\left[\mathrm{mm}^{2}\right]$ & $0.625 / 2.5$ & $\begin{array}{l}22.8, \\
\text { with void fraction }=0.8 \\
\text { due to static mixer }\end{array}$ \\
\hline Residence time $[\mathrm{sec}]$ & $0.29 / 4.3$ & 4.5 \\
\hline Linear velocity $[\mathrm{m} / \mathrm{sec}]$ & $0.45 / 0.11$ & 0.34 \\
\hline Reynolds number & $291 / 146$ & 1814 \\
\hline
\end{tabular}

efficient mixing could be assured while keeping the linear velocity in the same range as during the piloting tests. ${ }^{[8]}$ This resulted in a flowrate of $17 \mathrm{~mL} / \mathrm{min}$ in the lab experiments compared to a flow rate of $456 \mathrm{~mL} / \mathrm{min}$ on piloting scale. The main dimensions and process conditions of both scales are compared in Table 1. On both scales, the Reynolds number was in the laminar region. Whereas in the piloting scale the Reynolds number was on the upper limit of the laminar flow region $(\operatorname{Re}=1814)$, due to a much smaller channel diameter it was considerably lower in the laboratory conditions. The relatively low Reynolds number in the Ehrfeld reactor is compensated by microstructures that are producing mixing enhancing vortices on the lower edge of the laminar flow region (starting from $\mathrm{Re} \approx 100$ ).

\section{Fouling Study in the Ehrfeld Reactor: Development of Anti-fouling Strategy}

With a set-up as described in the sections above, a control experiment was established, where THF and HexLi solution were pumped at $15 \mathrm{~mL} / \mathrm{min}$ and $2 \mathrm{~mL} / \mathrm{min}$ respectively. The two streams were pre-cooled to $-30{ }^{\circ} \mathrm{C}$ and $0{ }^{\circ} \mathrm{C}$ respectively and then combined in the Ehrfeld reactor $\left(\mathrm{Tj}:-30{ }^{\circ} \mathrm{C}\right)$. Under these conditions a series of experiments with and without substrate were performed (Table 2). The reference point at $105 \mathrm{ppm}$ water in THF mixed with HexLi could be well reproduced three times, delivering a process time of about 25 min until plugging. The increase of water content to 158 and $238 \mathrm{ppm}$ led to slightly shorter run times (Table 2, entry 2 and 3 ) thus confirming the initial hypothesis.

In a next step, several methods of water removal were considered, tested and compared to each other. Thereby, the goal was to find a method that eventually can dry a substrate solution well below $50 \mathrm{ppm}$ :

1) In order to suppress the water content in THF, several hygroscopic inorganic salts along with an ion exchange resin were tested. These include magnesium sulfate, potassium hydroxide, sodium sulfate, calcium hydride, aluminum oxide and Dowex Marathon C. All of them failed to reduce the water level below 100 ppm.

2) Using distillation strategies, the THF/water azeotrope was removed in the distillate. While this approach failed in a onestage distillation, a rectification column was able to reduce water content below $35 \mathrm{ppm}$. It has to be noted that in practice it is difficult to determine lower water content as the sampling itself will lead to the addition of some moisture.

3) The use of $\mathrm{MgCl}_{2}$ was found partially beneficial in delaying the fouling, while the addition of inorganic salts aimed to improve the solubility of $\mathrm{LiOH}$ as mixed-salt did not significantly help (Table 2, entry 4 and 5).

4) A consistent reduction of the water content below $35 \mathrm{ppm}$ was obtained when the THF was treated with $3 \AA$, $4 \AA$ or $5 \AA$ hydrated molecular sieves. This method allowed prolonging the processing time up to $4 \mathrm{~h}$ with no fouling of the reactor (Table 2 , entry 6).

5) As an alternative to molecular sieves, a new chemical drying method based on the use of $i \mathrm{PrMgCl}$ was envisaged. In this case, a calculated amount (1.1 equiv. compared to the water present in the solution) of Grignard reagent was added to the THF. The chemical reaction of $i \mathrm{PrMgCl}$ with water, leading to the formation of inorganic magnesium salts and propane, allowed a water-free feedstock to be prepared. The resulting mixture was then filtered over a filter with a nominal pore size of $0.2 \mu \mathrm{m}$ to filter off inorganic magnesium salts. When the THF of this quality was pumped against HexLi a $4 \mathrm{~h}$ run without fouling of the reactor was achieved (Table 2, entry 7).

Table 2. Comparison of run time with different drying strategies

\begin{tabular}{|c|c|c|c|}
\hline Entry & Solution & KF (ppm) & Run time (min) \\
\hline 1 & THF & 105 & $25,30,30$ \\
\hline 2 & THF & 158 & 19 \\
\hline 3 & THF & 238 & 25 \\
\hline 4 & THF & $238+\mathrm{LiCl}$ & 25 \\
\hline 5 & THF & $238+\mathrm{MgCl}_{2}$ & 48 \\
\hline 6 & THF & 35 & $4 \mathrm{~h}$ no block \\
\hline 7 & THF & With $i \mathrm{PrMgCl}$ & $4 \mathrm{~h}$ no block \\
\hline 8 & $\begin{array}{c}\text { Substrate } 0.3 \mathrm{M} \\
\text { in THF }\end{array}$ & 180 & 65 \\
\hline 9 & $\begin{array}{c}\text { Substrate } 0.3 \mathrm{M} \\
\text { in THF }\end{array}$ & 350 & 30 \\
\hline 10 & $\begin{array}{c}\text { Substrate } 0.3 \mathrm{M} \\
\text { in THF }\end{array}$ & 50 & $4 \mathrm{~h}$ no block \\
\hline 11 & $\begin{array}{c}\text { Substrate } 0.3 \mathrm{M} \\
\text { in THF }\end{array}$ & With $i \mathrm{PrMgCl}$ & 4 h no block \\
\hline
\end{tabular}

Similar experiments were performed with the $0.3 \mathrm{M}$ solution of substrate (Table 2, entry 8 to 11). From the analysis of these data, it can be concluded that there is an inverse correlation between the amount of water present in the feedstock and the run time (Fig. 4).

Furthermore the presence of the substrate clearly extended the run time compared to the pure THF results. It is also worth saying that in the cases where fouling of the reactor occurred, it was always localized in the proximity of the mixing point, as most of the salt crush out quasi-instantaneously (Fig. 5). In the cases in which the water level in THF was reduced below 50 ppm by drying with molecular sieves or by addition of $i \mathrm{PrMgCl}$ the experiment did not lead to fouling of $\mathrm{LiOH}$ in the $4 \mathrm{~h}$ tested. 
Fig. 4. Graphical representation of Table 2.

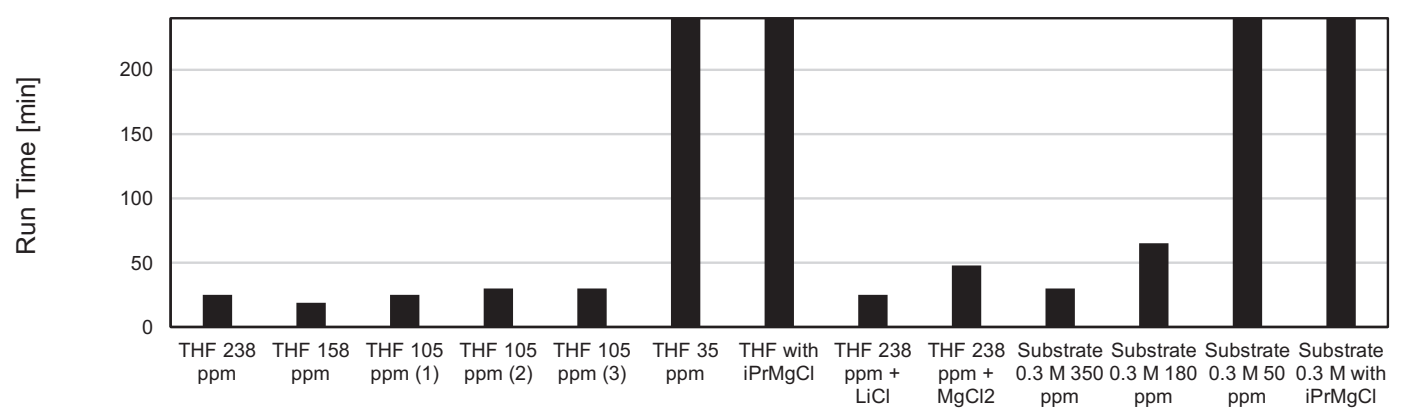

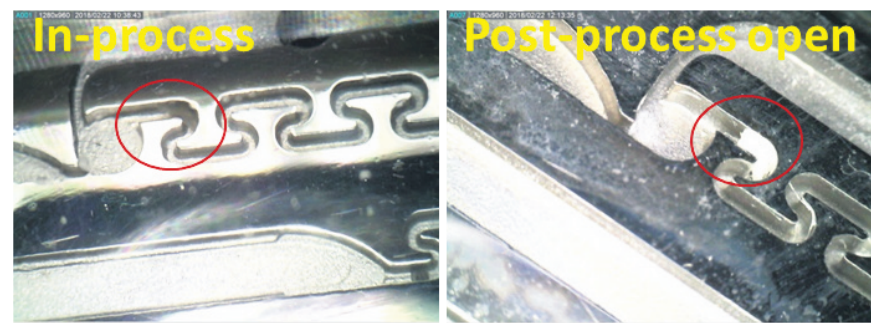

Fig. 5. Picture of the fouling observed while operating (left), reactor opened and dried (right).

\section{Long Runs in the Flow Lab Unit}

With the aim of further investigating the potential application at scale of the two most promising drying methods, i.e. drying by molecular sieves (method 4) and chemical drying by $i \mathrm{PrMgCl}$ (method 5) were tested in two long term runs of $30 \mathrm{~h}$. In this case, by using the set-up discussed above, the reaction of HexLi with THF was selected as model reaction. As shown before, this reference system has two advantages: on the one hand it fouls much faster than a system containing substrate, therefore pointing out any weakness of our drying methods much better (see Table 2). On the other hand side, the results obtained from these experiments should not only be applicable to the reaction described in this manuscript, but to all organolithium reactions. The first long run experiment was performed selecting the addition of $i \mathrm{PrMgCl}$ as the drying method. To further showcase the efficiency of this drying method, $50 \mathrm{~kg}$ of THF were intentionally spiked with water to a Karl-Fischer (KF) value of $383 \mathrm{ppm}$. This was followed by the addition of 1.1 equivalents of Grignard reagent, calculated based on the moles of water present in the solution. After stirring for $1 \mathrm{~h}$ at $20{ }^{\circ} \mathrm{C}$, the solution was discharged to a mobile tank via a 0.2 $\mu \mathrm{m}$ in-line filter to obtain a clear colorless solution. The mobile tank containing the freshly prepared solution was connected to the reactor unit and the experiment was performed for $30 \mathrm{~h}$ without pressure increase connected to fouling of the Ehrfeld reactor (Table 3 , entry 1 ).

In a similar way $50 \mathrm{~kg}$ of THF were added to $2.5 \mathrm{~kg}$ of $3 \AA$ molecular sieves. The resulting mixture was stirred at $20^{\circ} \mathrm{C}$ for $24 \mathrm{~h}$. After this time, a sample of the reaction mixture was analyzed with KF method indicating 38 ppm of water in the mixture. The mixture was then filtered and the output of the filtration was collected in a mobile tank which was used as feedstock for the flow experiment. Before starting the experiment a second sample was taken and analyzed with KF method indicating an uptake of water up to $58 \mathrm{ppm}$ during filtration and connection of the mobile tank to the reactor unit.

In this case, the reaction was performed for $9 \mathrm{~h}$ and $45 \mathrm{~min}$, until pressure increase due to fouling of the flow reactor was observed. To evaluate the reproducibility of the fouling phenomena, the reaction was performed again and in this case the reactor was operated for $8 \mathrm{~h}$ and $38 \mathrm{~min}$ (Table 3 , entry 2).

\section{Extension of Scope to other Solvents}

Subsequently, the chemical drying was tested in solvents which are commonly used in organolithium reactions. A series of experiments were performed using $\mathrm{PhCH}_{3}$ and Me-THF as solvents with and without Grignard addition. In particular, a standard quality of both solvents was initially tested with Karl Fischer analysis with added water to obtain 300 ppm solutions (Table 4, entry 1 and 2). In a similar fashion, after measuring the amount of water in MeTHF and $\mathrm{PhCH}_{3}$ and calculating the amount of $i \mathrm{PrMgCl}$ required, 1.1 equiv. compared to the moles of water in the solvent was added

Table 3. Long runs in flow lab unit

\begin{tabular}{|l|c|c|c|c|}
\hline Entry & Drying method & $\begin{array}{c}\text { Water content } \\
\text { before drying }\end{array}$ & $\begin{array}{c}\text { Water content } \\
\text { after drying }\end{array}$ & Run time \\
\hline 1 & Addition of $i \mathrm{PrMgCl}$ & $383 \mathrm{ppm}$ & $0 \mathrm{ppm}$ & $30 \mathrm{~h}$, no blockage occurred \\
\hline 2 & Molecular sieves & $100 \mathrm{ppm}$ & $58 \mathrm{ppm}$ & $\begin{array}{c}\text { Reactor fouling after } 9 \mathrm{~h} 45 \mathrm{~min}, \\
\text { reaction restarted } 8 \mathrm{~h} 38 \mathrm{~min}\end{array}$ \\
\hline
\end{tabular}

Table 4. Solvent scope

\begin{tabular}{|l|c|c|c|}
\hline Entry & Solution & KF (ppm) & Run time (min) \\
\hline 1 & $\mathrm{PhCH}_{3}$ & 300 & 12 \\
\hline 2 & $\mathrm{Me}-\mathrm{THF}$ & 300 & 15 \\
\hline 3 & $\mathrm{PhCH}_{3}$ & With $i \mathrm{PrMgCl}$ & 4 h no block \\
\hline 4 & $\mathrm{Me}-\mathrm{THF}$ & With $i \mathrm{PrMgCl}$ & 4 h no block \\
\hline 5 & $\mathrm{Me}-\mathrm{THF}$ & $\mathrm{MeMgCl}$ & 4 h no block \\
\hline
\end{tabular}




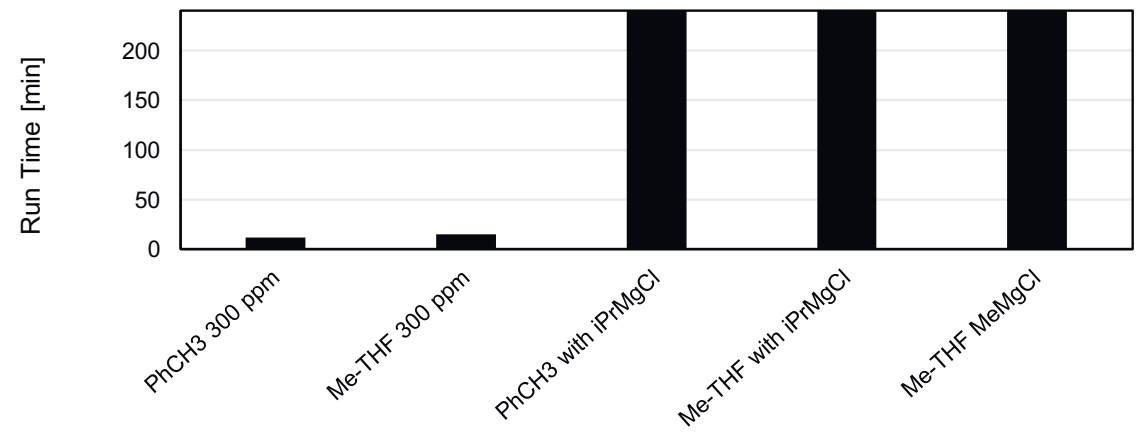

Fig. 6. Graphical representation of results reported in Table 4.

(Table 4 entry 3 and 4). The resulting mixture was filtered and the filtrate collected at the pump. It is worth mentioning that in the case of Me-THF and $\mathrm{PhCH}_{3}$, a slow filtration was observed compared to THF. As the results of these experiment, it can be noted that in all cases the addition of the drying agent $i \mathrm{PrMgCl}$ or $\mathrm{MeMgCl}$ extended the run time (Table 4 and Fig. 6).

It is worth mentioning that when choosing the drying agent, the chemical reactivity and compatibility with the substrate must be considered. Impact of the reagent on reaction outcome, formation of potential new impurities and additional safety investigations as well as the technical feasibility in the plant have to be evaluated case-bycase. In our experience, the reagent $i \mathrm{PrMgCl}$ was found as the best compromise between reactivity and technical implementation.

\section{Confirmation of Anti-fouling Strategy in Pilot Scale}

In a next step, the efficiency of chemical drying by Grignard addition was tested in the pilot plant. Therefore, the solvent of choice was THF as it was the solvent used in the campaign described initially. An impression of the pilot plant set-up is shown in Fig. 7. The tubular reactor was a Fluitec Contiplant system ${ }^{[10]}$ with helical static mixers (CSE-W) and X-type static mixers (CSE-X/4).

In addition to the chemical approach to extend run time two different designs of mixers were compared. In order to compare both mixers with regards to run time, two runs were conducted with commercial THF with about 60 ppm water, knowing that this concentration would already lead to plugging. The following mixers were selected:

- Run 1: X-type static mixer. The HexLi stream was pumped through a capillary into the center of a X-type static mixer, where it got mixed with the THF stream (Fig. 8);

- Run 2: Venturi mixer. The HexLi was injected through the same capillary as above. It was mixed with the THF stream at the entrance of the Venturi element (Fig. 9). This mixer forces the flow of THF and HexLi through a small diameter section $(\mathrm{ID}=2.1 \mathrm{~mm})$ to increase the velocity.

In run 1, a total runtime of $16 \mathrm{~h}$ was achieved (Fig. 10). A pressure increase was observed after $12 \mathrm{~h}$. The parallel trends on both the THF and the HexLi pumps indicated that the plugging is located inside the reactor at or after the mixing point. The run was stopped at a pressure of 4.5 bar and the HexLi was flushed out of the reactor using hexane (with THF pump running further).

In run 2 , the total runtime was $21.5 \mathrm{~h}$, until reaching a maximum pressure of 6 bar (Fig. 11). The pressure increase was already significant after $7 \mathrm{~h}$ in this case, but the regular drops in pressure of about 0.5 to 1 bar were observed, indicating that this type of mixer has the ability of cleaning itself to some limited extent.

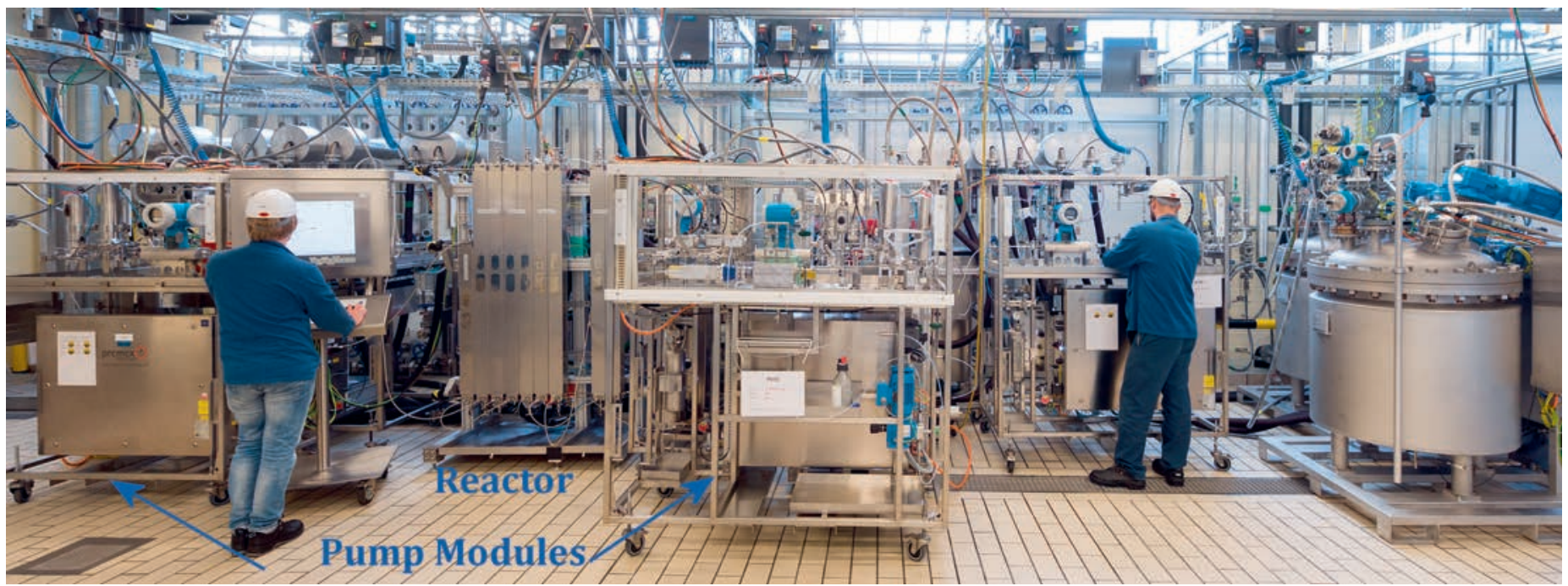

Fig. 7. Pilot plant equipment for verification of the chemical drying method.

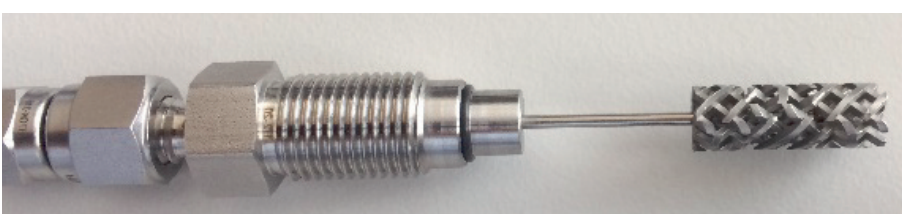

Fig. 8. X-type static mixer with capillary inlet tube.

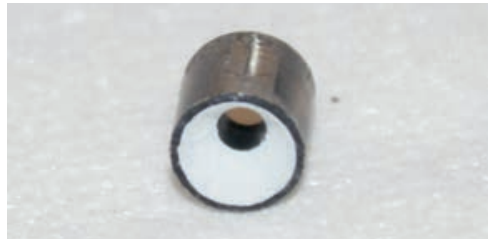

Fig. 9. Venturi mixer with ID $=2.1 \mathrm{~mm}$. 
Fig. 10. Run 1 with normal THF and X-type mixer: flow rate, pressure and density at the THF pump (P030) and the HexLi pump (P040). The experiment was stopped overnight and started up the next day (no or low flow between $15 \mathrm{~h}$ and $30 \mathrm{~h}$ ).

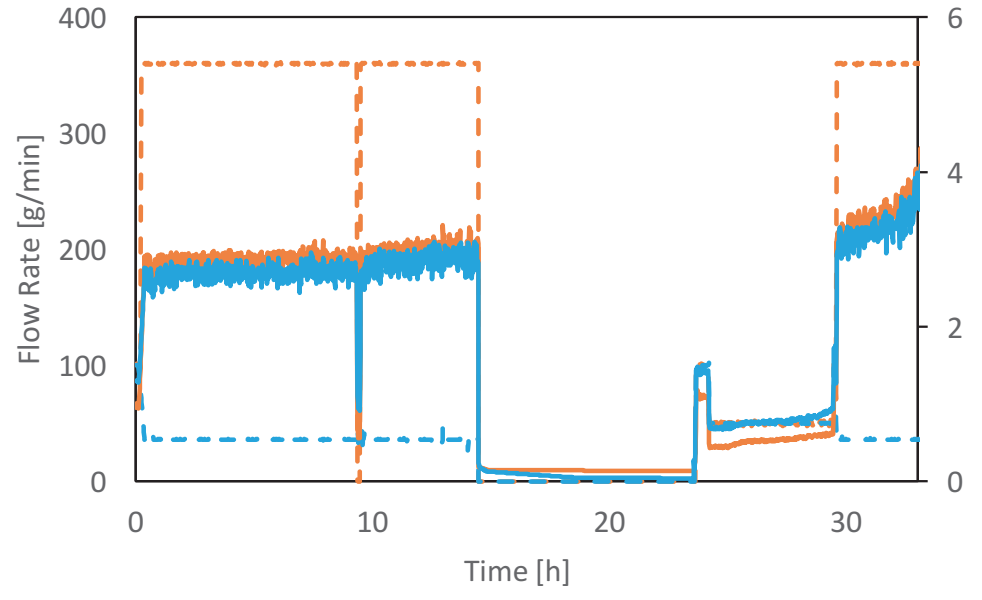

6

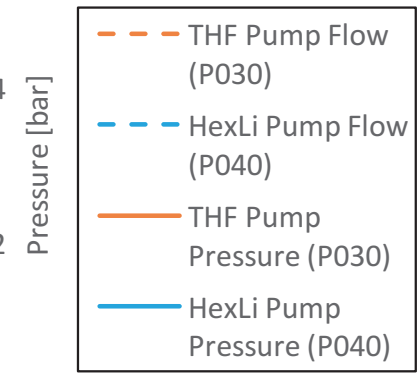

Fig. 11. Run 2 with normal THF and Venturi mixer: flow rate, pressure and density at the THF pump (P030) and the HexLi pump (P040). The experiment was stopped overnight and started up the next day (no flow between $10 \mathrm{~h}$ and $19 \mathrm{~h}$ ).

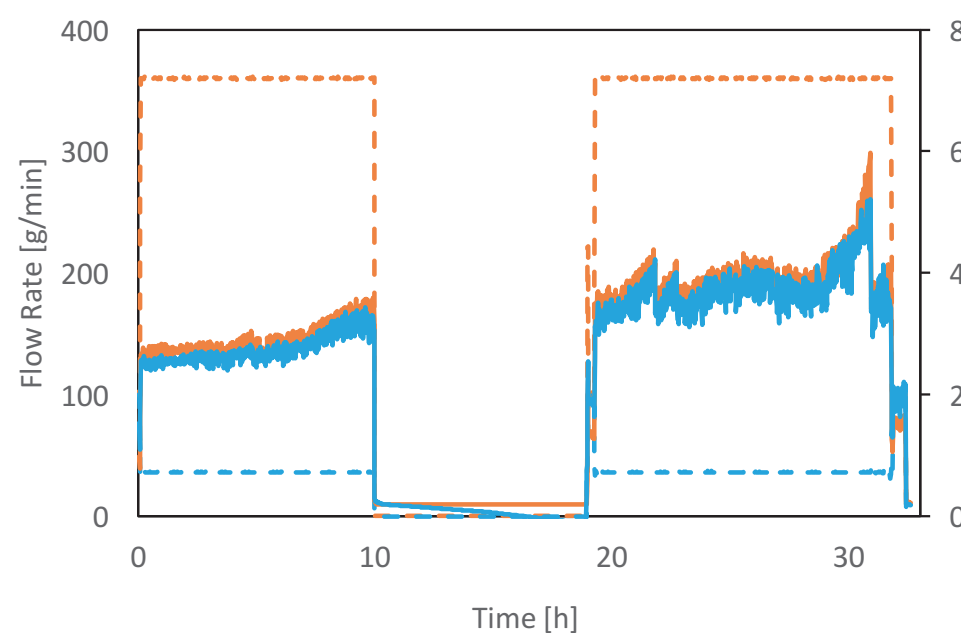

8

Time [h]

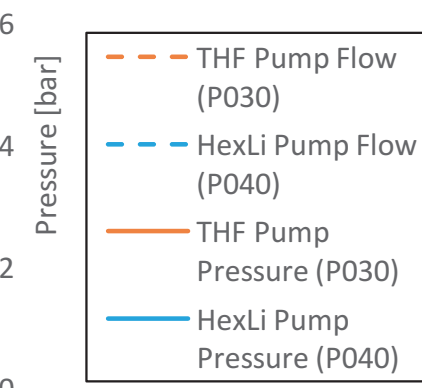

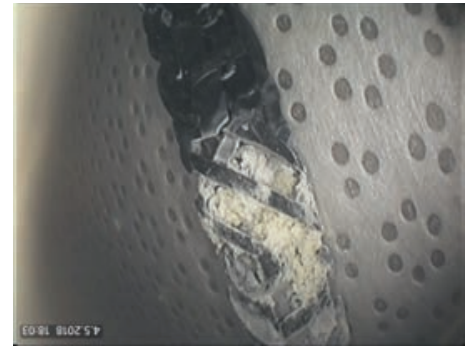

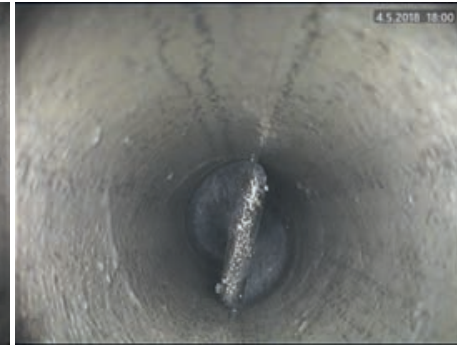

Fig. 12. Visual inspection after run 1. (left) X-type static mixer removed from tubular reactor; (right) Inner view of the tubular reactor with helical static mixer located after the mixer.

After each run, the reactor was opened for visual inspection (Figs 12 and 13). In both cases, significant amounts of solids were observed at the mixing point and downstream along the reactor wall and the helical static mixer. The solid accumulation was in line with the lab observations, i.e. the fouling of the reactor occurred by formation of insoluble $\mathrm{LiOH}$ at the mixing point between HexLi and THF and caused the pressure increase.

In a direct comparison of both mixer types, the Venturi mixer led to steep pressure rises much earlier in the process than the static mixer, i.e. after $12 \mathrm{~h}$. Due to its self-cleaning ability, eventually it was able to run for 10 more hours and outperform the static mixer, whose pressure rose steadily. For this reason, the Venturi mixer was selected for further studies. Nevertheless, it needs to be emphasized that more runs would be necessary to prove a clear advantage of one mixer compared to the other.

In order to clean the pre-coolers and the reactor after each run, a cleaning loop was built with an independent pump. The following cleaning sequence was performed at room temperature: 1) THF
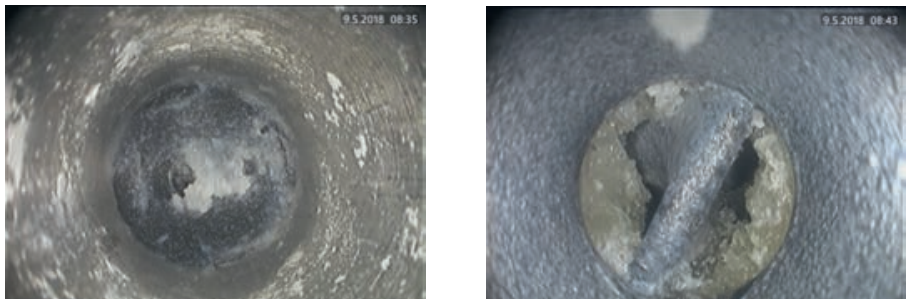

Fig. 13. Visual inspection after run 2. (left) Venturi-type static mixer inside the tubular reactor; (right) Inner view of the tubular reactor with helical static mixer located after the mixer.

was pumped through both pre-coolers and through the reactor to remove the hexane which remained from the flush of the HexLi line; 2$)$ the accumulated solid $(\mathrm{LiOH})$ was dissolved by pumping aqueous acetic acid; 3 ) the pre-coolers and the reactor were flushed back to THF until a sufficiently low water content was reached.

After the reference runs with 'normal' THF, the chemical drying method was tested at the pilot scale during a $30 \mathrm{~h}$ run (Fig. 14). $650 \mathrm{~kg}$ of THF were dried by addition of Grignard at 1.1 equivalent of the water content $(60 \mathrm{ppm})$, i.e $1.2 \mathrm{~kg}$ of $i \mathrm{PrMgCl}$ $2 \mathrm{M}$ solution. The entire run was performed without significant pressure increase. It was only interrupted due to a technical issue for about $1.5 \mathrm{~h}$.

The visual inspection of the pipes after flushing with THF at about $60 \mathrm{ppm}$ water (through THF pump) and hexane (through HexLi pump) revealed no accumulation of solid, but only a thin layer of white solid on some of the parts (Fig. 15). There is a clear improvement with the Grignard drying method compared to the solid accumulation observed in the shorter runs with the normal THF (undried), as shown in Figs 12 and 13. 


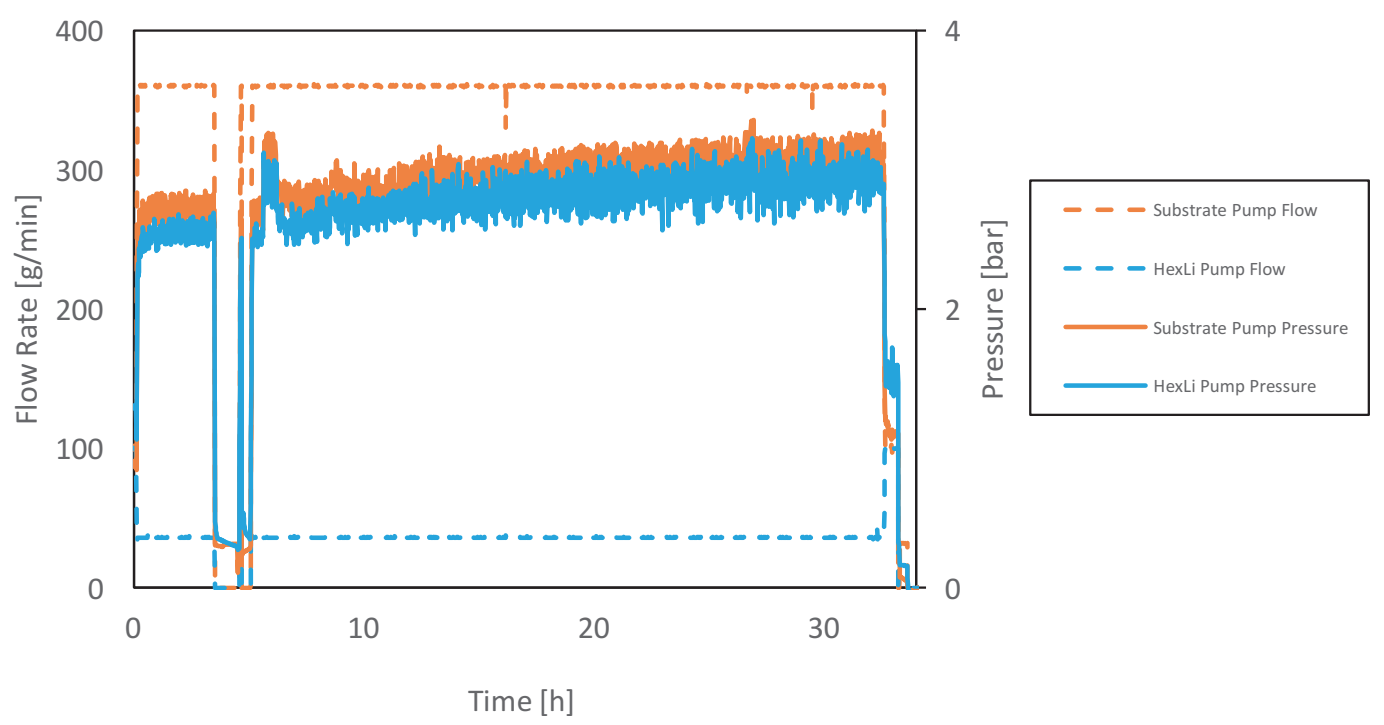

Fig. 14. $30 \mathrm{~h}$ run with Grignard dried THF: flow rate, pressure and density at the THF pump (P030) and the HexLi pump (P040).
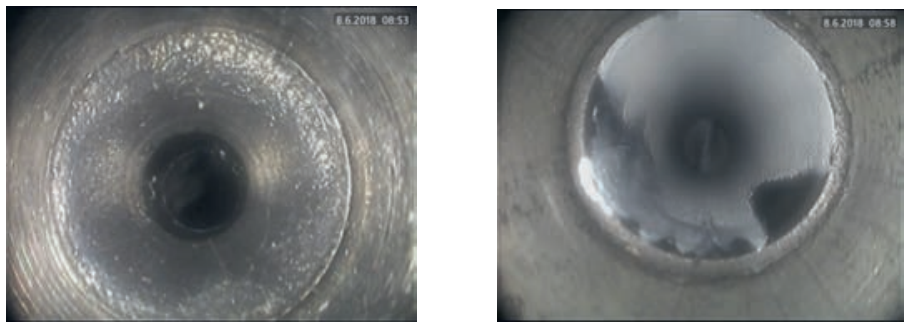

Fig. 15. Visual inspection after $30 \mathrm{~h}$ run with Grignard dried THF. (left) Venturi-type static mixer inside the tubular reactor; (right) Inner view of the tubular reactor with helical static mixer located after the mixer.

The $30 \mathrm{~h}$ run thus confirmed the efficiency of the chemical drying method, which avoids the accumulation of $\mathrm{LiOH}$ in the reactor and enables longer run time in the pilot plant, like in the lab.

\section{Conclusion}

In summary in the present work an efficient method to avoid fouling of flow reactors by formation of $\mathrm{LiOH}$ is reported and described. The addition of small amount of $i \mathrm{PrMgCl}$ to the feed solution to be reacted with HexLi has been beneficial to remove water and ensure an extended run time. The methodology has been showcased both in lab and plant, and the performance of undried and chemically dried solutions has been investigated. The methodology has been also successfully extended to other solvents which are potentially used in organolithium mediated reactions and to other potential drying agents. We are confident that the concept of drying the solvents with chemical reagents would work for practically any solvent used for these transformations and that many kinds of drying agents following the chemical drying criteria (e.g. alkali-, alkaline earth- and transition metal-bases, alkali and alkaline earth metals) are expected to produce similar results in avoid fouling. However, the choice of the drying agent and the application of this anti-fouling strategy have to be carefully evaluated. The balance between chemical reactivity of the substrate, technical feasibility (extra unit operations, e.g. filtration), complexity and run time expectation has to be evaluated case by case.

\section{Experimental}

All experiments at laboratory scale were performed using equipment and reagents purchased from commercial sources and used as received. The water content was determined by Karl Fischer analysis using a 917 Coulometer with generator electrode with diaphragm, Coulomat CG as catholyte and Coulomat AG as anolyte. The flow equipment, which was used for lab-scale experiments, has been built up as follows. Two micro annular pumps (HNP micro-gear pump) were used to separately pump the two feedstocks through a Coriolis mass-flow (Bronkhorst miniCORI-FLOW), a check valve, a pressure sensor (WIKA S-11) and SS filters (HNP Inline filter, $1 / 4$ inch). The two streams were directed to the Ehrfeld reactor, with the defined flow rate, precooled at the defined temperature, mixed and allowed to react at $-30{ }^{\circ} \mathrm{C}$. The outflow was quenched in a mixture of THF: $\mathrm{H}_{2} \mathrm{O}$ $8: 1$ cooled at $5^{\circ} \mathrm{C}$.

\section{Acknowledgements}

The authors are grateful to the operational team: Joerg Classen, Maurice Koenemann, Raphael Reinhard, Ricardo Pereira, Sebastien Schuehmacher, Herve Bohrer and Ingo Emme for the support during plant operation. We are also thankful to Kevin Braun, Pascale Hoehn, Marian Lanz and Jonathan Schmuck for the safety related investigations.

Received: June 18, 2019

[1] a) L. X. Yu, M. Kopcha, Int. J. Pharm. 2017, 528, 354, doi: 10.1016/j.ijpharm.2017.06.039; b) T. F. O'Connor, L. X. Yu, S. L. Lee. Int. J. Pharm. 2016, 509, 492, doi: 10.1016/j.ijpharm.2016.05.058.

[2] a) A. R. Bogdan, A. W. Dombrowski, J. Med. Chem. 2019, doi: 10.1021/acs. jmedchem.8b01760; b) B. Martin, H. Lehmann, H. Yang, L. Chen, X. Tian, J. Polenk, B. Schenkel, Curr. Opin. Green Sust. Chem. 2018, 11, 27, doi: 10.1016/j.cogsc.2018.03.005.

[3] a) A. C. Fisher, M. H. Kamga, C. Agarabi, K., Brorson, S. L. Lee, S. Yoon, Trends Biotechnol. 2019, 37, 253, doi: 10.1016/j.tibtech.2018.08.008; b) K. P. Cole, M. D. Johnson, Expert Rev. Clin. Pharmacol. 2018, 11, 5, doi: 10.1080/17512433.2018.1413936; c) C. L. Burcham, A. J. Florence, M. D. Johnson, Аnпи. Rev. Chem. Biomol. Eng. 2018, 9, 253, doi: 10.1146/ annurev-chembioeng-060817-084355; d) R. Gérardy, N. Emmanuel, T. Toupy, V.-E. Kassin, N. N. Tshibalonza, M. Schmitz, J.-C. M. Monbaliu, Eur. J. Org. Chem. 2018, 2301, doi: 10.1002/ejoc.201800149; e) J. Britton, S. Majumdar, G. A., Chem. Soc. Rev. 2018, 47, 5891, doi: 10.1039/c7cs00906b; f) D. E. Fitzpatrick, S. V. Ley, Tetrahedron 2018, 74, 3087, doi: 10.1016/j.tet.2017.08.050.

[4] For recent examples see: a) A. Nagaki, H. Yamashita, K. Hirose, Y. Tsuchihashi, J. Yoshida, Angew. Chem. Int. Ed. 2019, 58, 4027, doi: 10.1002/anie.201814088; b) A. Nagaki, K. Sasatsuki, S. Ishiuchi, N. Miuchi, M. Takumi, J. Yoshida, Chem. Eur. J. 2019, 25, 4946, doi: 10.1002/ chem.201900743; c) D. A. Thaisrivongs, J. R. Naber, N. J. Rogus, G. Spencer, Org. Process Res. Dev. 2018, 22, 403, doi: 10.1021/acs.oprd.7b00385; d) H. Zhang, S. L., Buchwald, J. Am. Chem. Soc. 2017, 139, 11590, doi: 10.1021/ jacs.7b06630; e) M. A. Ganiek, M. R. Becker, G. Berionni, H. Zipse, P. Knochel, Chem. Eur. J. 2017, 23, 10280, doi: 10.1002/chem.201702593.; f) A. Hafner, V. Mancino, M. Meisenbach, B. Schenkel, J. Sedelmeier, Org. Lett. 2017, 19, 786, doi: 10.1021/acs.orglett.6b03753; g) F. Susanne, B. Martin, M. Aubry, J. Sedelmeier, F. Lima, S. Sevinc, L. Piccioni, J. Haber, B. Schenkel, F. Venturoni, Org. Process Res. Dev. 2017, 21, 1779, doi: 10.1021/ acs.oprd.7b00254; h) A. Hafner, P. Filipponi, L. Piccioni, M. Meisenbach, B. Schenkel, F. Venturoni, J. Sedelmeier, Org. Process Res. Dev. 2016, 
20, 1833, doi: 10.1021/acs.oprd.6b00281; i) A. Hafner, M. Meisenbach, J. Sedelmeier, Org. Lett. 2016, 18, 3630, doi: 10.1021/acs.orglett.6b01681; j) J. Sedelmeier, F. Venturoni, Chim. Oggi 2014, 32, 26; k) J. Sedelmeier, F. Lima, A. Litzler, B. Martin, F. Venturoni, Org. Lett. 2013, 15, 5546, doi: 10.1021/ol402706a.

[5] 'Organometallic Flow Chemistry', Ed. T. Noël, Springer, Heidelberg, 2016.

[6] S. Laue, V. Haverkamp, L. Mleczko, Org. Process Res. Dev. 2016, 20, 480, doi: 10.1021/acs.oprd.5b00183.

[7] For clogging of continuous flow systems see for example: a) G. Giri, L. Yang, Y. Mo, K. F. Jensen, Cryst. Growth Des. 2019, 19, 98, doi: 10.1021/ acs.cgd.8b00999; b) M. Schoenitz, L. Grundemann, W. Augustin, S. Scholl,
Chem. Commun. 2015, 51, 8213, doi: 10.1039/C4CC07849G; c) R. L. Hartman, Org. Process Res. Dev. 2012, 16, 870, doi: 10.1021/op200348t; d) T. Noël, J. R. Naber, R. L. Hartman, J. P. McMullen, K. F. Jensen, S. L. Buchwald, Chem. Sci. 2011, 2, 287, doi: 10.1039/C0SC00524J; e) S. Kuhn,

T. Noël, L. Gu, P. L. Heider, K. F. Jensen, Lab Chip 2011, 11, 2488, doi: 10.1039/C1LC20337A.

[8] S. Schwolow, J. Hollmann, B. Schenkel, T. Röder, Org. Process Res. Dev. 2012, 16, 1513, doi: 10.1021/op300107z.

[9] https://ehrfeld.com/home.html, 2019.

[10] https://www.fluitec.ch/static-mixing-heat-exchange.html, 2019. 